\title{
NEW FORMS OF STRONG COMPACTNESS IN TERMS OF IDEALS
}

\author{
Néstor Raúl Pachón \\ Department of Mathematics \\ Colombian School of Engineering \\ AK 45 No. 205-59, 111166, Bogotá, COLOMBIA
}

\begin{abstract}
The aim of this paper is to introduce and study new types of strong compactness, modulo an ideal, called $\rho \mathcal{I}$-compactness and $\sigma \mathcal{I}$-compactness.

Several of their properties are presented and some effects of various kinds of functions on them are studied. We compare this new spaces with other known types of strong compactness modulo an ideal.
\end{abstract}

AMS Subject Classification: 54D30, 54C10

Key Words: ideal, $\mathcal{I}$-compact, $S \mathcal{I}$-compact, $\alpha \mathcal{I}$-compact, $\beta \mathcal{I}$-compact, $\gamma \mathcal{I}$-compact

\section{Introduction and Preliminaries}

This work is developed around the concept of compactness modulo an ideal, which was introduced by Newcomb [13], and investigated among others by Hamlett and Jancovic [6], Rancin [15] Gupta and Kaur [5]. Some classes of strong compactness modulo an ideal were studied by Newcomb [13], Hamlett et al. [7], Abad El Monsef [2], Nasef and Noiri [11], Nasef [12] and Hosny [8]. Also, some classes of weak compactness, module an ideal, were studied by Gupta and Noiri [4].

The purpose of this paper is to introduce and investigate the classes of $\rho \mathcal{I}$ compact and $\sigma \mathcal{I}$-compact spaces. The behavior of this spaces under certain kinds of functions also is investigated.

An ideal on a set $X$ is a nonempty family $\mathcal{I}$ of subsets of $X$ such that:

Received: October 6, 2015

Published: February 15, 2016 (c) 2016 Academic Publications, Ltd.

url: www.acadpubl.eu 
(i) If $B \in \mathcal{I}$ and $A \subseteq B \subseteq X$, then $A \in \mathcal{I}$.

(ii) If $A \in \mathcal{I}$ and $B \in \mathcal{I}$, then $A \cup B \in \mathcal{I}$.

For example, if $X$ is a set and $B \subseteq X$, then the following sets are ideals on $X$ :

$$
\mathcal{P}(B)=\{A \subseteq X: A \subseteq B\}, \quad \mathcal{I}_{f}=\{A \subseteq X: A \text { is finite }\}
$$

and

$$
\mathcal{I}_{c}=\{A \subseteq X: A \text { is countable }\} .
$$

If $(X, \tau)$ is a topological space and $\mathcal{I}$ is an ideal on $X$, then $(X, \tau, \mathcal{I})$ is called an ideal space.

A subset $A$ of a space $(X, \tau)$ is said to be $g$-closed [10] if $\bar{A} \subseteq U$ whenever $A \subseteq U$ and $U \in \tau$. It is clear that every closed set is g-closed, but the converse is not true.

If $(X, \tau, \mathcal{I})$ is an ideal space, $(Y, \beta)$ is a topological space and $f: X \rightarrow Y$ is a function, then $f(\mathcal{I})=\{f(I): I \in \mathcal{I}\}$ is an ideal on $Y[13]$.

If $(X, \tau, \mathcal{I})$ is an ideal space, the set $\mathcal{B}=\{U \backslash I: U \in \tau$ and $I \in \mathcal{I}\}$ is a base for a topology $\tau^{*}$, finer than $\tau$.

If $(X, \tau)$ is a topological space and $A \subseteq X$ then $\bar{A}$ and $\operatorname{int}(A)$ will, respectively, denote the closure and interior of $\mathrm{A}$ in $(X, \tau)$.

\section{2. $\rho \mathcal{I}$-compact spaces}

We recall that a subset $A$ of an ideal space $(X, \tau, \mathcal{I})$ is said to be $\mathcal{I}$-compact [13], if for every open cover $\left\{V_{\alpha}\right\}_{\alpha \in \Lambda}$ of $A$ by elements of $\tau$, there exists $\Lambda_{0} \subseteq \Lambda$, finite, such that $A \backslash \bigcup_{\alpha \in \Lambda_{0}} V_{\alpha} \in \mathcal{I}$. The ideal space $(X, \tau, \mathcal{I})$ is said to be $\mathcal{I}$-compact if $X$ is $\mathcal{I}$-compact.

It is clear that $(X, \tau)$ is compact if and only if $(X, \tau,\{\varnothing\})$ is $\{\varnothing\}$-compact, and that if $(X, \tau)$ is compact then $(X, \tau, \mathcal{I})$ is $\mathcal{I}$-compact.

Definition 2.1 If $(X, \tau, \mathcal{I})$ is an ideal space and $A \subseteq X, A$ is said to be $\rho \mathcal{I}$-compact if for every family $\left\{V_{\alpha}\right\}_{\alpha \in \Lambda}$ of open subsets of $X$, if $A \backslash \bigcup_{\alpha \in \Lambda} V_{\alpha} \in \mathcal{I}$ then there exists $\Lambda_{0} \subseteq \Lambda$, finite, such that $A \backslash \bigcup_{\alpha \in \Lambda_{0}} V_{\alpha} \in \mathcal{I}$. The ideal space $(X, \tau, \mathcal{I})$ is said to be $\rho \mathcal{I}$-compact if $X$ is $\rho \mathcal{I}$-compact.

It is clear that $(X, \tau)$ is compact if and only if $(X, \tau,\{\varnothing\})$ is $\rho\{\varnothing\}$-compact, and that if $(X, \tau, \mathcal{I})$ is $\rho \mathcal{I}$-compact then $(X, \tau, \mathcal{I})$ is $\mathcal{I}$-compact. The converse is not true. 
Example 2.1 If $X=[0,+\infty), \tau=\{\varnothing, X\} \cup\{(r,+\infty): r \geq 0\}$ and $\mathcal{I}=\mathcal{I}_{f}$, then:

i) $(X, \tau, \mathcal{I})$ is $\mathcal{I}$-compact, because if $\left\{V_{\alpha}\right\}_{\alpha \in \Lambda}$ is an open cover of $X$, then there exists $\alpha_{0} \in \Lambda$ with $V_{\alpha_{0}}=X$, and so $X \backslash V_{\alpha_{0}} \in \mathcal{I}$.

ii) $(X, \tau, \mathcal{I})$ is not $\rho \mathcal{I}$-compact, because $X \backslash \bigcup_{r>0}(r,+\infty)=\{0\} \in \mathcal{I}$, but if $n$ is a positive integer and $0<r_{1}<r_{2}<\cdots<r_{n}$, then $X \backslash \bigcup_{i=1}^{n}\left(r_{i},+\infty\right)=$ $X \backslash\left(r_{1},+\infty\right) \notin \mathcal{I}$.

In the example 3.1 we show a $\rho \mathcal{I}$-compact ideal space.

Definition 2.2 A subset $A$ of an ideal space $(X, \tau, \mathcal{I})$ is said to be $\mathcal{I} g$-closed if for every $U \in \tau$, if $A \backslash U \in \mathcal{I}$ then $\bar{A} \subseteq U$.

It is clear that $A$ is $\{\varnothing\} g$-closed if and only if $A$ is g-closed. We note that if $A$ is $\mathcal{I} g$-closed then $\mathrm{A}$ is g-closed. The converse is not true.

Example 2.2 1) If $X=\mathbb{R}, \tau=\{\varnothing, \mathbb{R}\} \cup\{(r,+\infty): r \in \mathbb{R}\}, \mathcal{I}=\{B: B \subseteq \mathbb{Q}\}$, and if $A=\mathbb{Q}$, then:

(a) $A$ is g-closed because if $U \in \tau$ and $A \subseteq U$, then $U=\mathbb{R}$ and so $\bar{A} \subseteq U$.

(b) $A$ is not $\mathcal{I} g$-closed since $A \backslash(0,+\infty) \in \mathcal{I}$, but $\bar{A}=\mathbb{R} \nsubseteq(0,+\infty)$.

2) If $X=\{0,1,2,3\}, \tau=\{\varnothing,\{0\},\{1\},\{0,1\}, X\}, \mathcal{I}=\{\varnothing,\{0\},\{1\},\{0,1\}\}$ and $A=\{2\}$, then $A$ is $\mathcal{I} g$-closed because if $U \in \tau$ and $A \backslash U \in \mathcal{I}$, we have that $A \subseteq U$, and so $U=X$ and $\bar{A} \subseteq U$.

Theorem 2.1 If $(X, \tau, \mathcal{I})$ is an ideal space and $\mathcal{B}$ is a base for $\tau$, then $(X, \tau, \mathcal{I})$ is $\rho \mathcal{I}$-compact if and only if for all family $\left\{V_{\alpha}\right\}_{\alpha \in \Lambda}$ of open sets in $\mathcal{B}$, if $X \backslash \bigcup_{\alpha \in \Lambda} V_{\alpha} \in \mathcal{I}$ then there exists $\Lambda_{0} \subseteq \Lambda$, finite, with $X \backslash \bigcup_{\alpha \in \Lambda_{0}} V_{\alpha} \in \mathcal{I}$.

Proof. $(\rightarrow)$ It is obvious.

$(\leftarrow)$ Let $\left\{V_{\alpha}\right\}_{\alpha \in \Lambda}$ be a family of non-empty open subsets of $X$ such that $X \backslash \bigcup_{\alpha \in \Lambda} V_{\alpha} \in \mathcal{I}$. For all $\alpha \in \Lambda$ there exists a family $\left\{W_{\alpha \beta}: \beta \in \Lambda_{\alpha}\right\}$ of elements in $\mathcal{B}$ such that $V_{\alpha}=\bigcup_{\beta \in \Lambda_{\alpha}} W_{\alpha \beta}$.

Given that $X \backslash \bigcup_{\alpha \in \Lambda} \bigcup_{\beta \in \Lambda_{\alpha}} W_{\alpha \beta} \in \mathcal{I}$ and $(X, \tau, \mathcal{I})$ is $\rho \mathcal{I}$-compact, there exist $W_{\alpha_{1} \beta_{1}}, W_{\alpha_{2} \beta_{2}}, \ldots, W_{\alpha_{r} \beta_{r}}$ such that $X \backslash \bigcup_{i=1}^{r} W_{\alpha_{i} \beta_{i}} \in \mathcal{I}$. But $X \backslash \bigcup_{i=1}^{r} V_{\alpha_{i}} \subseteq$ $X \backslash \bigcup_{i=1}^{r} W_{\alpha_{i} \beta_{i}}$, and so $X \backslash \bigcup_{i=1}^{r} V_{\alpha_{i}} \in \mathcal{I}$. 
Theorem 2.2 If $(X, \tau, \mathcal{I})$ is an ideal space, then the following statements are equivalents:

(a) $(X, \tau, \mathcal{I})$ is $\rho \mathcal{I}$-compact.

(b) $\left(X, \tau^{*}, \mathcal{I}\right)$ is $\rho \mathcal{I}$-compact.

(c) For any family $\left\{F_{\alpha}\right\}_{\alpha \in \Lambda}$ of closed subsets of $X$, if $\bigcap_{\alpha \in \Lambda} F_{\alpha} \in \mathcal{I}$ then there exists $\Lambda_{0} \subseteq \Lambda$, finite, such that $\bigcap_{\alpha \in \Lambda_{0}} F_{\alpha} \in \mathcal{I}$.

Proof. It is clear that $(a) \leftrightarrow(c)$. Since $\tau \subseteq \tau^{*}$ we have that $(b) \rightarrow(a)$.

$(a) \rightarrow(b)$ We apply the Theorem 2.1. The set $\mathcal{B}=\{U \backslash I: U \in \tau$ and $I \in \mathcal{I}\}$ is a base for $\tau^{*}$.

Let $\left\{V_{\alpha}\right\}_{\alpha \in \Lambda}$ be a family of open subsets in $\mathcal{B}$ with $X \backslash \bigcup_{\alpha \in \Lambda} V_{\alpha} \in \mathcal{I}$. For all $\alpha \in \Lambda$ there exist $W_{\alpha} \in \tau$ and $I_{\alpha} \in \mathcal{I}$ such that $V_{\alpha}=W_{\alpha} \backslash I_{\alpha}$. Since $X \backslash \bigcup_{\alpha \in \Lambda} W_{\alpha} \in \mathcal{I}$, there exists $\Lambda_{0} \subseteq \Lambda$, finite, such that $X \backslash \bigcup_{\alpha \in \Lambda_{0}} W_{\alpha} \in \mathcal{I}$. Now $X \backslash \bigcup_{\alpha \in \Lambda_{0}} V_{\alpha} \subseteq\left(X \backslash \bigcup_{\alpha \in \Lambda_{0}} W_{\alpha}\right) \cup \bigcup_{\alpha \in \Lambda_{0}} I_{\alpha} \in \mathcal{I}$, and so $X \backslash \bigcup_{\alpha \in \Lambda_{0}} V_{\alpha} \in \mathcal{I}$.

Thus $\left(X, \tau^{*}, \mathcal{I}\right)$ is $\rho \mathcal{I}$-compact.

Next we study the behavior of some types of subspaces of a $\rho \mathcal{I}$-compact space.

Theorem 2.3 If $(X, \tau, \mathcal{I})$ is $\rho I$-compact and $A \subseteq X$ is $\mathcal{I} g$-closed, then $A$ is $\rho \mathcal{I}$-compact.

Proof. Let $\left\{V_{\alpha}\right\}_{\alpha \in \Lambda}$ be a family of open subsets of $X$ such that $A \backslash \bigcup_{\alpha \in \Lambda} V_{\alpha} \in$ $\mathcal{I}$. Since $\mathrm{A}$ is $\mathcal{I} g$-closed, $\bar{A} \subseteq \bigcup_{\alpha \in \Lambda} V_{\alpha}$. Then $X=(X \backslash \bar{A}) \cup \bigcup_{\alpha \in \Lambda} V_{\alpha}$ and so $X \backslash\left[(X \backslash \bar{A}) \cup \bigcup_{\alpha \in \Lambda} V_{\alpha}\right]=\varnothing \in \mathcal{I}$. Given that $X$ is $\rho \mathcal{I}$-compact, there exists $\Lambda_{0} \subseteq \Lambda$, finite, such that $X \backslash \bigcup_{\alpha \in \Lambda_{0}} V_{\alpha} \in \mathcal{I}$ or $X \backslash\left[(X \backslash \bar{A}) \cup \bigcup_{\alpha \in \Lambda_{0}} V_{\alpha}\right] \in \mathcal{I}$. In any case $X \backslash\left[(X \backslash \bar{A}) \cup \bigcup_{\alpha \in \Lambda_{0}} V_{\alpha}\right] \in \mathcal{I}$. But $X \backslash\left[(X \backslash \bar{A}) \cup \bigcup_{\alpha \in \Lambda_{0}} V_{\alpha}\right]=\bar{A} \backslash \bigcup_{\alpha \in \Lambda_{0}} V_{\alpha}$, and since $A \backslash \bigcup_{\alpha \in \Lambda_{0}} V_{\alpha} \subseteq \bar{A} \backslash \bigcup_{\alpha \in \Lambda_{0}} V_{\alpha} \in \mathcal{I}$, we have that $A \backslash \bigcup_{\alpha \in \Lambda_{0}}^{\alpha \in \Lambda_{0}} V_{\alpha} \in \mathcal{I}$.

Theorem 2.4 If $A$ and $B$ are $\rho \mathcal{I}$-compact subsets of an ideal space $(X, \tau, \mathcal{I})$, then $A \cup B$ is $\rho \mathcal{I}$-compact.

Proof. Let $\left\{V_{\alpha}\right\}_{\alpha \in \Lambda}$ be a family of open subsets such that $(A \cup B) \backslash \bigcup_{\alpha \in \Lambda} V_{\alpha} \in$ $\mathcal{I}$. Since $A \backslash \bigcup_{\alpha \in \Lambda} V_{\alpha} \subseteq(A \cup B) \backslash \bigcup_{\alpha \in \Lambda} V_{\alpha}$ and $B \backslash \bigcup_{\alpha \in \Lambda} V_{\alpha} \subseteq(A \cup B) \backslash \bigcup_{\alpha \in \Lambda} V_{\alpha}$ then 
$A \backslash \bigcup_{\alpha \in \Lambda} V_{\alpha} \in \mathcal{I}$ and $B \backslash \bigcup_{\alpha \in \Lambda} V_{\alpha} \in \mathcal{I}$, and so there exist $\Lambda_{1} \subseteq \Lambda$ and $\Lambda_{2} \subseteq \Lambda$, finite, with $A \backslash \bigcup_{\alpha \in \Lambda_{1}} V_{\alpha} \in \mathcal{I}$ and $B \backslash \bigcup_{\alpha \in \Lambda_{2}} V_{\alpha} \in \mathcal{I}$. This implies that $A \backslash \bigcup_{\alpha \in \Lambda_{1} \cup \Lambda_{2}} V_{\alpha} \in \mathcal{I}$ and $B \backslash \bigcup_{\alpha \in \Lambda_{1} \cup \Lambda_{2}} V_{\alpha} \in \mathcal{I}$, and so $(A \cup B) \backslash \bigcup_{\alpha \in \Lambda_{1} \cup \Lambda_{2}} V_{\alpha} \in \mathcal{I}$.

Theorem 2.5 Let $(X, \tau, \mathcal{I})$ be an ideal space and $A \subseteq X$. Suppose that for all $U \in \tau$, if $A \backslash U \in \mathcal{I}$ then there exists $B \subseteq X$ such that $B$ is $\rho \mathcal{I}$-compact, $A \subseteq B$ and $B \backslash U \in \mathcal{I}$. Then $A$ is $\rho \mathcal{I}$-compact.

Proof. Let $\left\{V_{\alpha}\right\}_{\alpha \in \Lambda}$ be a family of open subsets of $X$ such that $A \backslash \bigcup_{\alpha \in \Lambda} V_{\alpha} \in$ I. There exists $B \subseteq X$ such that $B$ is $\rho \mathcal{I}$-compact, $A \subseteq B$ and $B \backslash \bigcup_{\alpha \in \Lambda}^{\alpha \in \Lambda} V_{\alpha} \in$ $\mathcal{I}$. There exists $\Lambda_{0} \subseteq \Lambda$, finite, with $B \backslash \bigcup_{\alpha \in \Lambda_{0}} V_{\alpha} \in \mathcal{I}$. Since $A \backslash \bigcup_{\alpha \in \Lambda_{0}}^{\alpha \in \Lambda} V_{\alpha} \subseteq$ $B \backslash \bigcup_{\alpha \in \Lambda_{0}} V_{\alpha}$ we have that $A \backslash \bigcup_{\alpha \in \Lambda_{0}} V_{\alpha} \in \mathcal{I}$.

Theorem 2.6 If $(X, \tau, \mathcal{I})$ is an ideal space, $A \subseteq B \subseteq X, B \subseteq \bar{A}$ and $A$ is $\mathcal{I}$ $g$-closed then $A$ is $\rho \mathcal{I}$-compact if and only if $B$ is $\rho \mathcal{I}$-compact.

Proof. $(\rightarrow)$ Let $\left\{V_{\alpha}\right\}_{\alpha \in \Lambda}$ be a family of open subsets of $X$ such that $B \backslash \bigcup_{\alpha \in \Lambda} V_{\alpha} \in \mathcal{I}$. Then $A \backslash \bigcup_{\alpha \in \Lambda} V_{\alpha} \in \mathcal{I}$, and given that $A$ is $\rho \mathcal{I}$-compact there exists $\Lambda_{0} \subseteq \Lambda$, finite, such that $A \backslash \bigcup_{\alpha \in \Lambda_{0}} V_{\alpha} \in \mathcal{I}$. Since $A$ is $\mathcal{I}$ g-closed, $\bar{A} \subseteq \bigcup_{\alpha \in \Lambda_{0}} V_{\alpha}$, and so $\bar{A} \backslash \bigcup_{\alpha \in \Lambda_{0}} V_{\alpha} \in \mathcal{I}$. This implies $B \backslash \bigcup_{\alpha \in \Lambda_{0}} V_{\alpha} \in \mathcal{I}$.

$(\leftarrow)$ Let $\left\{V_{\alpha}\right\}_{\alpha \in \Lambda}$ be a family of open subsets of $X$ such that $A \backslash \bigcup_{\alpha \in \Lambda} V_{\alpha} \in \mathcal{I}$. Given that $A$ is $\mathcal{I}$ g-closed, $\bar{A} \backslash \bigcup_{\alpha \in \Lambda} V_{\alpha}=\varnothing \in \mathcal{I}$, and this implies $B \backslash \bigcup_{\alpha \in \Lambda} V_{\alpha} \in \mathcal{I}$. Since $B$ is $\rho \mathcal{I}$-compact, there exists $\Lambda_{0} \subseteq \Lambda$, finite, with $B \backslash \bigcup_{\alpha \in \Lambda_{0}} V_{\alpha} \in \mathcal{I}$. Hence $A \backslash \bigcup_{\alpha \in \Lambda_{0}} V_{\alpha} \in \mathcal{I}$.

The following theorem is consequence of [Theorem 2.2, [5]].

Theorem 2.7 Let $(X, \tau, \mathcal{I})$ be an ideal space such that $(X, \tau)$ is $T_{2}$. If $A$ is a $\rho \mathcal{I}$-compact subset of $X$, then $A$ is closed in $\left(X, \tau^{*}\right)$.

Theorem 2.8 Let $(X, \tau, \mathcal{I})$ be a $\rho \mathcal{I}$-compact space such that $(X, \tau)$ is $T_{2}$. If $F$ and $G$ are disjoint $\mathcal{I} g$-closed subsets of $X$, then there exist disjoint open subsets $U$ and $V$ of $X$, such that $F \backslash U \in \mathcal{I}$ and $G \backslash V \in \mathcal{I}$. 
Proof. The result is clear if $F=\varnothing$ or if $G=\varnothing$. Suppose that $F \neq \varnothing$ and $G \neq \varnothing$.

Theorem 2.3 implies that $F$ and $G$ are $\rho \mathcal{I}$-compact subsets of $X$.

We choose $g \in G$, arbitrary but fixed.

For all $f \in F$ there exist disjoint $U_{f} \in \tau$ and $V_{f} \in \tau$ such that $f \in U_{f}$ and $g \in V_{f}$. Given that $F \backslash \bigcup_{f \in F} U_{f}=\varnothing \in \mathcal{I}$, there exists $F_{g} \subseteq F$, finite, with $F \backslash \bigcup_{f \in F_{g}} U_{f} \in \mathcal{I}$. Let $T_{g}=\bigcup_{f \in F_{g}} U_{f}$ and $W_{g}=\bigcap_{f \in F_{g}} V_{f}$. It is clear that $T_{g} \cap W_{g}=\varnothing$.

Now, since that $G \backslash \bigcup_{g \in G} W_{g}=\varnothing \in \mathcal{I}$ and $G$ is $\rho I$-compact, there exists $G_{0} \subseteq G$, finite, such that $G \backslash \bigcup_{g \in G_{0}} W_{g} \in \mathcal{I}$.

Let $U=\bigcap_{g \in G_{0}} T_{g}$ and $V=\bigcup_{g \in G_{0}} W_{g}$. We note that $F \backslash U=\bigcup_{g \in G_{0}}\left(F \backslash T_{g}\right) \in \mathcal{I}$ and $G \backslash V \in \mathcal{I}$.

Moreover, $U \cap V=\varnothing$, because if $u \in U \cap V$ then there exists $g_{1} \in G_{0}$ with $u \in W_{g_{1}}$ and, since $u \in T_{g_{1}}$, we have that $T_{g_{1}} \cap W_{g_{1}} \neq \varnothing$, contradiction.

Now we study the behavior of $\rho \mathcal{I}$-compactness under certain types of functions.

Theorem 2.9 If $(X, \tau, \mathcal{I})$ is $\rho \mathcal{I}$-compact, $f:(X, \tau) \rightarrow(Y, \beta)$ is a continuous function and if $\mathcal{J}=\left\{B \subseteq Y: f^{-1}(B) \in \mathcal{I}\right\}$ then:

(1) $\mathcal{J}$ is an ideal on $Y$.

(2) $(Y, \beta, \mathcal{J})$ is $\rho \mathcal{J}$-compact.

Proof. (1) Suppose that $A \subseteq B \subseteq Y$ and $B \in \mathcal{J}$. Since $f^{-1}(A) \subseteq f^{-1}(B) \in$ $\mathcal{I}$, then $f^{-1}(A) \in \mathcal{I}$, and so $A \in \mathcal{J}$.

Now, if $A \in \mathcal{J}$ and $B \in \mathcal{J}$, then $f^{-1}(A) \in \mathcal{I}$ and $f^{-1}(B) \in \mathcal{I}$, and then $f^{-1}(A \cup B)=f^{-1}(A) \cup f^{-1}(B) \in \mathcal{I}$. This implies that $A \cup B \in \mathcal{J}$.

(2) Let $\left\{V_{\alpha}\right\}_{\alpha \in \Lambda}$ be a family of open subsets of $Y$ such that $Y \backslash \bigcup_{\alpha \in \Lambda} V_{\alpha} \in \mathcal{J}$.

Since $X \backslash \bigcup_{\alpha \in \Lambda} f^{-1}\left(V_{\alpha}\right)=f^{-1}\left(Y \backslash \bigcup_{\alpha \in \Lambda} V_{\alpha}\right) \in \mathcal{I}$ and $(X, \tau, \mathcal{I})$ is $\rho \mathcal{I}$-compact, there exists $\Lambda_{0} \subseteq \Lambda$, finite, with $f^{-1}\left(Y \backslash \bigcup_{\alpha \in \Lambda_{0}} V_{\alpha}\right)=X \backslash \bigcup_{\alpha \in \Lambda_{0}} f^{-1}\left(V_{\alpha}\right) \in \mathcal{I}$. Thus $Y \backslash \bigcup_{\alpha \in \Lambda_{0}} V_{\alpha} \in \mathcal{J}$.

Theorem 2.10 If $(X, \tau, \mathcal{I})$ is $\rho \mathcal{I}$-compact and $f:(X, \tau) \rightarrow(Y, \beta)$ is a biyective continuous function, then $(Y, \beta, f(\mathcal{I}))$ is $\rho f(\mathcal{I})$-compact. 
Proof. Let $\left\{V_{\alpha}\right\}_{\alpha \in \Lambda}$ be a family of open subsets of $Y$ such that $Y \backslash \bigcup_{\alpha \in \Lambda} V_{\alpha} \in$ $f(\mathcal{I})$. There exists $I \in \mathcal{I}$ with $Y \backslash \bigcup_{\alpha \in \Lambda} V_{\alpha}=f(I)$. Then $I=f^{-1}(f(I))=$ $X \backslash \bigcup_{\alpha \in \Lambda} f^{-1}\left(V_{\alpha}\right) \in \mathcal{I}$. Given that $(X, \tau, \mathcal{I})$ is $\rho \mathcal{I}$-compact, there exists $\Lambda_{0} \subseteq \Lambda$, finite, with $f^{-1}\left(Y \backslash \bigcup_{\alpha \in \Lambda_{0}} V_{\alpha}\right)=X \backslash \bigcup_{\alpha \in \Lambda_{0}} f^{-1}\left(V_{\alpha}\right) \in \mathcal{I}$. Thus $Y \backslash \bigcup_{\alpha \in \Lambda_{0}} V_{\alpha}=$ $f\left(f^{-1}\left(Y \backslash \bigcup_{\alpha \in \Lambda_{0}} V_{\alpha}\right)\right) \in f(\mathcal{I})$.

If $f: X \rightarrow Y$ is an inyective function and $\mathcal{J}$ is an ideal on $Y$, then the set $f^{-1}(\mathcal{J})=\left\{f^{-1}(J): J \in \mathcal{J}\right\}$ is an ideal on $X[13]$.

Theorem 2.11 If $f:(X, \tau) \rightarrow(Y, \beta)$ is a biyective open function and $(Y, \beta, \mathcal{J})$ is $\rho \mathcal{J}$-compact, then $\left(X, \tau, f^{-1}(\mathcal{J})\right)$ is $\rho f^{-1}(\mathcal{J})$-compact.

Proof. Let $\left\{V_{\alpha}\right\}_{\alpha \in \Lambda}$ be a family of open subsets of $X$ such that $X \backslash \bigcup_{\alpha \in \Lambda} V_{\alpha} \in$ $f^{-1}(\mathcal{J})$. There exists $J \in \mathcal{J}$ with $X \backslash \bigcup_{\alpha \in \Lambda} V_{\alpha}=f^{-1}(J)$. Then $Y \backslash \bigcup_{\alpha \in \Lambda} f\left(V_{\alpha}\right)=$ $f\left(f^{-1}(J)\right)=J \in \mathcal{J}$, and given that $(Y, \beta, \mathcal{J})$ is $\rho \mathcal{J}$-compact then there exists $\Lambda_{0} \subseteq \Lambda$, finite, with $f\left(X \backslash \bigcup_{\alpha \in \Lambda_{0}} V_{\alpha}\right)=Y \backslash \bigcup_{\alpha \in \Lambda_{0}} f\left(V_{\alpha}\right) \in \mathcal{J}$. This implies that $X \backslash \bigcup_{\alpha \in \Lambda_{0}} V_{\alpha} \in f^{-1}(\mathcal{J})$.

\section{3. $\sigma \mathcal{I}$-compact spaces}

In this section we present a strong form of $\rho \mathcal{I}$-compactness. Some properties of these spaces are also presented.

Definition 3.1 If $(X, \tau, \mathcal{I})$ is an ideal space and $A \subseteq X, A$ is said to be $\sigma \mathcal{I}$ compact if for every family $\left\{V_{\alpha}\right\}_{\alpha \in \Lambda}$ of open subsets of $X$, if $A \backslash \bigcup_{\alpha \in \Lambda} V_{\alpha} \in \mathcal{I}$ then there exists $\Lambda_{0} \subseteq \Lambda$, finite, such that $A \subseteq \bigcup_{\alpha \in \Lambda_{0}} V_{\alpha}$. The ideal space $(X, \tau, \mathcal{I})$ is said to be $\sigma \mathcal{I}$-compact if $X$ is $\sigma \mathcal{I}$-compact.

We note that if $(X, \tau, \mathcal{I})$ is an ideal space and $\left(X, \tau^{*}, \mathcal{I}\right)$ is $\sigma \mathcal{I}$-compact, then $(X, \tau, \mathcal{I})$ is $\sigma \mathcal{I}$-compact, and that $(X, \tau, \mathcal{I})$ is $\sigma \mathcal{I}$-compact if and only if 
for any family $\left\{F_{\alpha}\right\}_{\alpha \in \Lambda}$ of closed subsets of $X$, if $\bigcap_{\alpha \in \Lambda} F_{\alpha} \in \mathcal{I}$ then there exists $\Lambda_{0} \subseteq \Lambda$, finite, such that $\bigcap_{\alpha \in \Lambda_{0}} F_{\alpha}=\varnothing$.

It is clear that $(X, \tau)$ is compact if and only if $(X, \tau,\{\varnothing\})$ is $\sigma\{\varnothing\}$-compact, and that if $(X, \tau, \mathcal{I})$ is $\sigma \mathcal{I}$-compact then $(X, \tau, \mathcal{I})$ is $\rho \mathcal{I}$-compact, and $(X, \tau)$ is compact.

Example 3.1 (1) Let $X=\mathbb{Z}^{+}, \tau=\{A \subseteq X: X \backslash A$ is finite $\} \cup\{\varnothing\}$ and $\mathcal{I}$ $=\mathcal{I}_{f}$. Then:

(a) The ideal space $(X, \tau, \mathcal{I})$ is $\rho \mathcal{I}$-compact, because if $\left\{F_{i}\right\}_{i \in \Lambda}$ is a family of closed subsets of $X$ with $\bigcap_{i \in \Lambda} F_{i} \in \mathcal{I}$, then there exists $i_{0} \in \Lambda$ such that $F_{i_{0}} \neq X$. Thus $F_{i_{0}} \in \mathcal{I}$.

(b) The ideal space $(X, \tau, \mathcal{I})$ is not $\sigma \mathcal{I}$-compact, because if $F_{n}=\{1,2, \ldots, n\}$ then $F_{n}$ is a closed subset of $X$ and $\bigcap_{n=1}^{\infty} F_{n}=\{1\} \in \mathcal{I}$, but if $n_{1}, n_{2}, \ldots, n_{r} \in \mathbb{Z}^{+}$ then $\bigcap_{k=1}^{r} F_{n_{k}} \neq \varnothing$.

(2) If $\tau$ is the usual topology for $\mathbb{R}$, and $\mathcal{I}=\{A: A \subseteq \mathbb{R}\}$, then $(\mathbb{R}, \tau)$ is not compact, but $(\mathbb{R}, \tau, \mathcal{I})$ is, evidently, $\rho \mathcal{I}$-compact.

(3) If $\tau$ is the usual topology for $X=[0,1]$ and $\mathcal{I}=\{\varnothing,\{1\}\}$, then $(X, \tau)$ is compact but $(X, \tau, \mathcal{I})$ is not $\rho \mathcal{I}$-compact. In fact, if $V_{n}=[0,1-1 / n)$, for all integer number $n \geq 2$, then $X \backslash \bigcup_{n \geq 2} V_{n}=\{1\} \in \mathcal{I}$, but if $k \in \mathbb{Z}^{+}$and $n_{1}, n_{2}, \ldots, n_{k}$ are integer numbers with $2 \leq n_{i}$, for all $i \in\{1,2, \ldots, k\}$, then $X \backslash \bigcup_{i=1}^{k} V_{n_{i}} \notin \mathcal{I}$.

In the example 4.1 we show a $\sigma \mathcal{I}$-compact ideal space.

Theorem 3.1 If $(X, \tau, \mathcal{I})$ is an ideal space and $\mathcal{B}$ is a base for $\tau$, then $(X, \tau, \mathcal{I})$ is $\sigma \mathcal{I}$-compact if and only if for every family $\left\{V_{\alpha}\right\}_{\alpha \in \Lambda}$ of ope sets in $\mathcal{B}$, if $X \backslash \bigcup_{\alpha \in \Lambda} V_{\alpha} \in \mathcal{I}$ then there exists $\Lambda_{0} \subseteq \Lambda$, finite, such that $X=\bigcup_{\alpha \in \Lambda_{0}} V_{\alpha}$.

Proof. $(\rightarrow)$ It is obvious.

$(\leftarrow)$ Let $\left\{V_{\alpha}\right\}_{\alpha \in \Lambda}$ be a family of non-empty open subsets of $X$ such that $X \backslash \bigcup_{\alpha \in \Lambda} V_{\alpha} \in \mathcal{I}$. For all $\alpha \in \Lambda$ there exists a family $\left\{W_{\alpha \beta}: \beta \in \Lambda_{\alpha}\right\}$ of elements in $\mathcal{B}$ such that $V_{\alpha}=\bigcup_{\beta \in \Lambda} W_{\alpha \beta}$. 
Given that $X \backslash \bigcup_{\alpha \in \Lambda} \bigcup_{\beta \in \Lambda_{\alpha}} W_{\alpha \beta} \in \mathcal{I}$ and $(X, \tau, \mathcal{I})$ is $\sigma \mathcal{I}$-compact, there exist $W_{\alpha_{1} \beta_{1}}, W_{\alpha_{2} \beta_{2}}, \ldots, W_{\alpha_{r} \beta_{r}}$ such that $X=\bigcup_{i=1}^{r} W_{\alpha_{i} \beta_{i}}$. But $X=\bigcup_{i=1}^{r} W_{\alpha_{i} \beta_{i}} \subseteq$ $\bigcup_{i=1}^{r} V_{\alpha_{i}} \subseteq X$, and so $X=\bigcup_{i=1}^{r} V_{\alpha_{i}}$

Next we study the behavior of some types of subspaces of a $\sigma \mathcal{I}$-compact space.

Theorem 3.2 If $(X, \tau, \mathcal{I})$ is $\sigma \mathcal{I}$-compact and $A \subseteq X$ is $\mathcal{I} g$-closed, then $A$ is $\sigma \mathcal{I}$-compact.

Proof. Let $\left\{V_{\alpha}\right\}_{\alpha \in \Lambda}$ be a family of open subsets of $X$ such that $A \backslash \bigcup_{\alpha \in \Lambda} V_{\alpha} \in$ $\mathcal{I}$. Since $\mathrm{A}$ is $\mathcal{I} g$-closed, $\bar{A} \subseteq \bigcup_{\alpha \in \Lambda} V_{\alpha}$. Then $X=(X \backslash \bar{A}) \cup \bigcup_{\alpha \in \Lambda} V_{\alpha}$ and so $X \backslash\left[(X \backslash \bar{A}) \cup \bigcup_{\alpha \in \Lambda} V_{\alpha}\right]=\varnothing \in \mathcal{I}$. Given that $X$ is $\sigma \mathcal{I}$-compact, there exists $\Lambda_{0} \subseteq \Lambda$, finite, such that $X=(X \backslash \bar{A}) \cup \bigcup_{\alpha \in \Lambda_{0}} V_{\alpha}$. Then $A=A \cap[(X \backslash \bar{A}) \cup$ $\left.\bigcup_{\alpha \in \Lambda_{0}} V_{\alpha}\right]=A \cap \bigcup_{\alpha \in \Lambda_{0}} V_{\alpha} \subseteq \bigcup_{\alpha \in \Lambda_{0}} V_{\alpha}$

Theorem 3.3 If $A$ and $B$ are $\sigma \mathcal{I}$-compact subsets of an ideal space $(X, \tau, \mathcal{I})$, then $A \cup B$ is $\sigma \mathcal{I}$-compact.

Proof. Let $\left\{V_{\alpha}\right\}_{\alpha \in \Lambda}$ be a family of open subsets such that $(A \cup B) \backslash \bigcup_{\alpha \in \Lambda} V_{\alpha} \in$ $\mathcal{I}$. Since $A \backslash \bigcup_{\alpha \in \Lambda} V_{\alpha} \subseteq(A \cup B) \backslash \bigcup_{\alpha \in \Lambda} V_{\alpha}$ and $B \backslash \bigcup_{\alpha \in \Lambda} V_{\alpha} \subseteq(A \cup B) \backslash \bigcup_{\alpha \in \Lambda} V_{\alpha}$ then $A \backslash \bigcup_{\alpha \in \Lambda} V_{\alpha} \in \mathcal{I}$ and $B \backslash \bigcup_{\alpha \in \Lambda} V_{\alpha} \in \mathcal{I}$, and so there exist $\Lambda_{1} \subseteq \Lambda$ and $\Lambda_{2} \subseteq \Lambda$, finite, such that $A \subseteq \bigcup_{\alpha \in \Lambda_{1}} V_{\alpha}$ and $B \subseteq \bigcup_{\alpha \in \Lambda_{2}} V_{\alpha}$. This implies that $A \subseteq \bigcup_{\alpha \in \Lambda_{1} \cup \Lambda_{2}} V_{\alpha}$ and $B \subseteq \bigcup_{\alpha \in \Lambda_{1} \cup \Lambda_{2}} V_{\alpha}$, and so $A \cup B \subseteq \bigcup_{\alpha \in \Lambda_{1} \cup \Lambda_{2}} V_{\alpha}$

Theorem 3.4 Let $(X, \tau, \mathcal{I})$ be an ideal space and $A \subseteq X$. Suppose that for all $U \in \tau$, if $A \backslash U \in \mathcal{I}$ then there exists $B \subseteq X$ such that $B$ is $\sigma \mathcal{I}$-compact, $A \subseteq B$ and $B \backslash U \in \mathcal{I}$. Then $A$ is $\sigma \mathcal{I}$-compact.

Proof. Let $\left\{V_{\alpha}\right\}_{\alpha \in \Lambda}$ be a family of open subsets of $X$ such that $A \backslash \bigcup V_{\alpha} \in$ $\mathcal{I}$. There exists $B \subseteq X$ such that $B$ is $\sigma \mathcal{I}$-compact, $A \subseteq B$ and $B \backslash \bigcup_{\alpha \in \Lambda}^{\alpha \in \Lambda} V_{\alpha} \in \mathcal{I}$. There exists $\Lambda_{0} \subseteq \Lambda$, finite, with $B \subseteq \bigcup_{\alpha \in \Lambda_{0}} V_{\alpha}$, and so $A \subseteq \bigcup_{\alpha \in \Lambda_{0}} V_{\alpha}$. 
Theorem 3.5 If $(X, \tau, \mathcal{I})$ is an ideal space, $A \subseteq B \subseteq X$ and $B \subseteq \bar{A}$ then:

(1) If $A$ is g-closed and $\sigma \mathcal{I}$-compact, then $B$ is $\sigma \mathcal{I}$-compact.

(2) If $A$ is $\mathcal{I} g$-closed and $B$ is $\sigma \mathcal{I}$-compact, then $A$ is $\sigma \mathcal{I}$-compact.

Proof. (1) Let $\left\{V_{\alpha}\right\}_{\alpha \in \Lambda}$ be a family of open subsets of $X$ such that $B \backslash \bigcup_{\alpha \in \Lambda} V_{\alpha} \in$ $\mathcal{I}$. Then $A \backslash \bigcup_{\alpha \in \Lambda} V_{\alpha} \in \mathcal{I}$, and given that $A$ is $\sigma \mathcal{I}$-compact, there exists $\Lambda_{0} \subseteq \Lambda$, finite, such that $A \subseteq \bigcup_{\alpha \in \Lambda_{0}} V_{\alpha}$. Since $A$ is g-closed, $\bar{A} \subseteq \bigcup_{\alpha \in \Lambda_{0}} V_{\alpha}$, and this implies $B \subseteq \bigcup_{\alpha \in \Lambda_{0}} V_{\alpha}$

(2) Let $\left\{V_{\alpha}\right\}_{\alpha \in \Lambda}$ be a family of open subsets of $X$ such that $A \backslash \bigcup_{\alpha \in \Lambda} V_{\alpha} \in \mathcal{I}$. Given that $A$ is $\mathcal{I}$ g-closed, $\bar{A} \backslash \bigcup_{\alpha \in \Lambda} V_{\alpha}=\varnothing \in \mathcal{I}$, and this implies $B \backslash \bigcup_{\alpha \in \Lambda} V_{\alpha} \in \mathcal{I}$. Since $B$ is $\sigma \mathcal{I}$-compact, there exists $\Lambda_{0} \subseteq \Lambda$, finite, with $B \subseteq \bigcup_{\alpha \in \Lambda_{0}} V_{\alpha}$. Hence $A \subseteq \bigcup_{\alpha \in \Lambda_{0}} V_{\alpha}$

The following theorem is consequence of Theorem 2.7.

Theorem 3.6 Let $(X, \tau, \mathcal{I})$ be an ideal space such that $(X, \tau)$ is $T_{2}$. If $A$ is a $\sigma \mathcal{I}$-compact subset of $X$, then $A$ is closed in $\left(X, \tau^{*}\right)$.

Now we study the behavior of $\sigma \mathcal{I}$-compactness under certain types of functions.

Theorem 3.7 If $(X, \tau, \mathcal{I})$ is $\sigma \mathcal{I}$-compact, $f:(X, \tau) \rightarrow(Y, \beta)$ is a continuous suryective function and if $\mathcal{J}=\left\{B \subseteq Y: f^{-1}(B) \in \mathcal{I}\right\}$ then $(Y, \beta, \mathcal{J})$ is $\sigma \mathcal{J}$-compact.

Proof. In the proof of Theorem 2.9 we show that $\mathcal{J}$ is an ideal on $Y$. Let $\left\{V_{\alpha}\right\}_{\alpha \in \Lambda}$ be a family of open subsets of $Y$ such that $Y \backslash \bigcup_{\alpha \in \Lambda} V_{\alpha} \in \mathcal{J}$. Since $X \backslash \bigcup_{\alpha \in \Lambda} f^{-1}\left(V_{\alpha}\right)=f^{-1}\left(Y \backslash \bigcup_{\alpha \in \Lambda} V_{\alpha}\right) \in \mathcal{I}$ and $(X, \tau, \mathcal{I})$ is $\sigma \mathcal{I}$-compact, there exists $\Lambda_{0} \subseteq \Lambda$, finite, such that $X=\bigcup_{\alpha \in \Lambda_{0}} f^{-1}\left(V_{\alpha}\right)$. Given that $f$ is suryective we have $Y=\bigcup_{\alpha \in \Lambda_{0}} V_{\alpha}$.

Theorem 3.8 If $(X, \tau, \mathcal{I})$ is $\sigma \mathcal{I}$-compact and $f:(X, \tau) \rightarrow(Y, \beta)$ is a biyective continuous function, then $(Y, \beta, f(\mathcal{I}))$ is $\sigma f(\mathcal{I})$-compact. 
Proof. Let $\left\{V_{\alpha}\right\}_{\alpha \in \Lambda}$ be a family of open subsets of $Y$ such that $Y \backslash \bigcup_{\alpha \in \Lambda} V_{\alpha} \in$ $f(\mathcal{I})$. There exists $I \in \mathcal{I}$ with $Y \backslash \bigcup_{\alpha \in \Lambda} V_{\alpha}=f(I)$. Then $I=f^{-1}(f(I))=$ $X \backslash \bigcup_{\alpha \in \Lambda} f^{-1}\left(V_{\alpha}\right) \in \mathcal{I}$. Given that $(X, \tau, \mathcal{I})$ is $\sigma \mathcal{I}$-compact, there exists $\Lambda_{0} \subseteq \Lambda$, finite, with $X=\bigcup_{\alpha \in \Lambda_{0}} f^{-1}\left(V_{\alpha}\right)$. Since $f$ is suryective, $Y=\bigcup_{\alpha \in \Lambda_{0}} V_{\alpha}$.

Theorem 3.9 If $f:(X, \tau) \rightarrow(Y, \beta)$ is a biyective and open function, and $(Y, \beta, \mathcal{J})$ is a $\sigma \mathcal{J}$-compact space, then $\left(X, \tau, f^{-1}(\mathcal{J})\right)$ is $\sigma f^{-1}(\mathcal{J})$-compact.

Proof. Let $\left\{V_{\alpha}\right\}_{\alpha \in \Lambda}$ be a family of open subsets of $X$ such that $X \backslash \bigcup_{\alpha \in \Lambda} V_{\alpha} \in$ $f^{-1}(\mathcal{J})$. There exists $J \in \mathcal{J}$ with $X \backslash \bigcup_{\alpha \in \Lambda} V_{\alpha}=f^{-1}(J)$. Then $Y \backslash \bigcup_{\alpha \in \Lambda} f\left(V_{\alpha}\right)=$ $f\left(f^{-1}(J)\right)=J \in \mathcal{J}$, and given that $(Y, \beta, \mathcal{J})$ is $\sigma \mathcal{J}$-compact then there exists $\Lambda_{0} \subseteq \Lambda$, finite, with $Y=\bigcup_{\alpha \in \Lambda_{0}} f\left(V_{\alpha}\right)$. This implies that $X=\bigcup_{\alpha \in \Lambda_{0}} V_{\alpha}$.

\section{4. $\rho \mathcal{I}$-compactness and $\sigma \mathcal{I}$-compactness are new strong forms of $\mathcal{I}$-compactness}

Other types of strong compactness modulo an ideal have been previously defined: $\beta \mathcal{I}$-compactness, $\gamma \mathcal{I}$-compactness, $S \mathcal{I}$-compactness, and $\alpha \mathcal{I}$-compactness.

It is known that $\gamma \mathcal{I}$-compactness $\Rightarrow \beta \mathcal{I}$-compactness $\Rightarrow S \mathcal{I}$-compactness $\Rightarrow$ $\alpha \mathcal{I}$-compactness $\Rightarrow \mathcal{I}$-compactness.

In this section we show that $\rho \mathcal{I}$-compactness and $\sigma \mathcal{I}$-compactness are really new classes of strong $\mathcal{I}$-compactness.

We recall that if $(X, \tau)$ is a topological space and $A \subseteq X$, then:

1) $A$ is said to be $\alpha$-open [14] if $A \subseteq \operatorname{int}(\overline{\operatorname{int}(A)})$.

2) $A$ is said to be $\beta$ - open [1] if $A \subseteq \overline{\operatorname{int}(\bar{A})}$.

3) $A$ is said to be $\gamma$-open [3] if $A \subseteq \operatorname{int}(\overline{\operatorname{int}(A)}) \cup \overline{\operatorname{int}(\bar{A})}$

4) $A$ is said to be semi -open [9], or simply $S$-open, if $A \subseteq \overline{\operatorname{int}(A)}$.

$5)$ If $(X, \tau, \mathcal{I})$ is an ideal space, and if $z \in\{\alpha, \beta, \gamma, S\}$ then $A$ is said to be $z \mathcal{I}$-compact if for every cover $\left\{V_{i}\right\}_{i \in \Lambda}$ of $A$ by $z$-open subsets of $X$, there exists $\Lambda_{0} \subseteq \Lambda$, finite, with $X \backslash \bigcup_{i \in \Lambda_{0}} V_{i} \in \mathcal{I}$. The space $(X, \tau, \mathcal{I})$ is said to be $z \mathcal{I}$-compact if $X$ is $z \mathcal{I}$-compact. 
The concepts of $\alpha \mathcal{I}$-compactness, $\beta \mathcal{I}$-compactness, $\gamma \mathcal{I}$-compactness and $S \mathcal{I}$-compactness are due to Nasef and Noiri [11], Hosny [8], Nasef [12] and Abd El-Monsef et al. [2], respectively.

The following example shows that, for all $z \in\{\alpha, \beta, \gamma, S\}, \sigma \mathcal{I}$-compactness $\nRightarrow z \mathcal{I}$-compactness, and then $\rho \mathcal{I}$-compactness $\nRightarrow z \mathcal{I}$-compactness.

Example 4.1 Suppose that $X=\mathbb{R}^{+}, \tau=\left\{\varnothing, X, X \backslash \mathbb{Z}^{+}\right\} \cup\left\{V_{n}: n \in \mathbb{Z}^{+}\right\}$, where $V_{n}=\bigcup_{k=0}^{n-1}(k, k+1)$, and $\mathcal{I}=\{A: A \subseteq(0,1)\}$. Then we have that:

(1) The ideal space $(X, \tau, \mathcal{I})$ is $\sigma \mathcal{I}$-compact.

If $\left\{U_{\alpha}\right\}_{\alpha \in \Lambda}$ is a family of open subsets of $X$ such that $X \backslash \bigcup_{\alpha \in \Lambda} U_{\alpha} \in \mathcal{I}$, there exists $\alpha_{0} \in \Lambda$ with $1 \in U_{\alpha_{0}}$, and so $U_{\alpha_{0}}=X$.

(2) $(X, \tau, \mathcal{I})$ is not $\alpha \mathcal{I}$-compact.

If $A_{n}=(0, n)$, for all $n \in \mathbb{Z}^{+}$, then $\operatorname{int}\left(A_{n}\right)=A_{n} \backslash\left(A_{n} \cap \mathbb{Z}^{+}\right), \overline{\operatorname{int}\left(A_{n}\right)}=$ $X, \operatorname{int}\left(\overline{\operatorname{int}\left(A_{n}\right)}\right)=X$ and so $A_{n}$ is $\alpha$-open. $\mathcal{I}$.

Moreover, $X=\bigcup_{n=1}^{\infty} A_{n}$ but if $n_{1}, n_{2}, \ldots, n_{m} \in \mathbb{Z}^{+}$we have that $X \backslash \bigcup_{k=1}^{m} A_{n_{k}} \notin$

Finally, we show an example of a $\gamma \mathcal{I}$-compact space which is not $\sigma \mathcal{I}$ compact. This implies that, for all $z \in\{\alpha, \beta, \gamma, S\}, z \mathcal{I}$-compactness $\nRightarrow \sigma \mathcal{I}$ compactness.

Example 4.2 Let $\tau$ be the topology on $\mathbb{Z}$ given by:

$V \in \tau$ if and only if, for all $n \in \mathbb{Z}$, if $n \in V$ then $[n]_{2} \in V$, where $[n]_{2}=0$ if $n$ is even, and $[n]_{2}=1$ if $n$ is odd.

Let $\mathcal{I}=\{A: A \subseteq \mathbb{Z} \backslash\{0,1\}\}$. We have that:

(1) $(\mathbb{Z}, \tau, \mathcal{I})$ is not $\sigma \mathcal{I}$-compact, because $(\mathbb{Z}, \tau)$ is not compact.

$\mathbb{Z}=\bigcup_{n=1}^{\infty}\{0,1,-n, n\}$ and $\{0,1,-n, n\} \in \tau$, for all $n \geq 1$.

(2) $B \subseteq \mathbb{Z}$ is $\gamma$-open if and only if $B$ is open.

It is clear that if $B$ is open, then $B$ is $\gamma$-open.

Suppose that $B$ is not open. There exists $N \in B$ with $[N]_{2} \notin B$.

If $N$ is even and $1 \in B$ then $\operatorname{int}(\overline{\operatorname{int}(B)})=2 \mathbb{Z}+1=\overline{\operatorname{int}(\bar{B})}$, where $2 \mathbb{Z}+1=\{2 n+1: n \in \mathbb{Z}\}$.

If $N$ is even and $1 \notin B$ then $\operatorname{int}(\overline{\operatorname{int}(B)})=\varnothing=\overline{\operatorname{int}(\bar{B})}$. 
If $N$ is odd and $0 \in B$ then $\operatorname{int}(\overline{\operatorname{int}(B)})=2 \mathbb{Z}=\overline{\operatorname{int}(\bar{B})}$, where $2 \mathbb{Z}=$ $\{2 n: n \in \mathbb{Z}\}$.

If $N$ is odd and $0 \notin B$ then $\operatorname{int}(\overline{\operatorname{int}(B)})=\varnothing=\overline{\operatorname{int}(\bar{B})}$.

Thus $B$ is not $\gamma$-open.

(3) $(\mathbb{Z}, \tau, \mathcal{I})$ is $\gamma \mathcal{I}$-compact.

If $\left\{B_{i}\right\}_{i \in \Lambda}$ is an open cover of $\mathbb{Z}$, there exist $i_{0} \in \Lambda$ and $i_{1} \in \Lambda$, such that $0 \in B_{i_{0}}$ and $1 \in B_{i_{1}}$. Then $\mathbb{Z} \backslash\left(B_{i_{0}} \cup B_{i_{1}}\right) \in \mathcal{I}$.

Question. There is a $\gamma \mathcal{I}$-compact space $(X, \tau, \mathcal{I})$ which is not $\rho \mathcal{I}$-compact?

\section{References}

[1] M. E. Abd El-Monsef, S. N. El Deeb and R.A. Mahmoud, $\beta$-open sets and $\beta$-continuous mappings, Bull. Fac. Sci. Assiut Univ., 12 (1983), 77-90.

[2] M. E. Abd El-Monsef, E. F. Lashien and A. A. Nasef, S-compactness via ideals, Tamkang J. Math., 24, No. 4 (1993), 431-443.

[3] A. A. El Atik, A study of some types of mappings on topological spaces, Master's thesis, Faculty of Science, Tanta University, Tanta, Egypt, (1997).

[4] M. K. Gupta and T. Noiri, C-compactness modulo an ideal, International J. Math. and Math. Sci., 2006, (2006), 1-12. DOI: 10.1155/IJMMS/2006/78135

[5] A. Gupta and R. Kaur, Compact spaces with respect to an ideal, International. J. P. and Ap. Math., 92, No. 3 (2014), 443-448. DOI: 10.12732/ijpam.v92i3.11

[6] T. R. Hamlett and D. Jancović, Compactness with respect to an ideal, Boll. Un. Math. Ital., 7, No. 4B (1990), 849-861.

[7] T. R. Hamlett, D. Jancović and D. Rose, Countable compactness with respect to an ideal, Math. Chronicle, 20, (1991), 109-126.

[8] R. A. Hosny, Some types of compactness via ideal, International J. Sci. \& Eng. Res., 4, No. 5 (2013), 1293-1296.

[9] N. Levine, Semi-open and semi-continuity in topological spaces, Amer. Math. Mountly, 70, (1963), 36-41. DOI: 10.2307/2312781

[10] N. Levine, Generalized closed sets in topological spaces, Rend. Circ. Mat. Palermo, 19, (1970), 89-96.

[11] A. A. Nasef and T. Noiri, On $\alpha$-compact modulo an ideal, Far East J. Math. Sci., 6, No. 6 (1998), 857-865.

[12] A. A. Nasef, Some classes of compactness in terms of ideals, Soochow Jour. of Math., 27, No. 3 (2001), 343-352.

[13] R. L. Newcomb, Topologies which are compact modulo an ideal, Ph. Dissertation, Univ. of Cal. at Santa Barbara, (1967).

[14] O. Njastad, On some classes of nearly open sets, Pacific J. Math., 15, (1965), 961-970. DOI: $10.2140 /$ pjm.1965.15.961

[15] D. V. Rancin, Compactness modulo an ideal, Soviet Math. Dokl., 13, No. 1 (1972), 193-197. 
